\title{
Multidisciplinary Approach to Unravelling the Relative Contribution of Different Oxylipins in Indirect Defense of Arabidopsis thaliana
}

\author{
Tjeerd A. L. Snoeren • Remco M. P. Van Poecke • \\ Marcel Dicke
}

Received: 28 July 2009/Revised: 11 September 2009/Accepted: 16 September 2009/Published online: 2 October 2009

(C) The Author(s) 2009. This article is published with open access at Springerlink.com

\begin{abstract}
The oxylipin pathway is commonly involved in induced plant defenses, and is the main signal-transduction pathway induced by insect folivory. Herbivory induces the production of several oxylipins, and consequently alters the so-called 'oxylipin signature' in the plant. Jasmonic acid (JA), as well as pathway intermediates are known to induce plant defenses. Indirect defense against herbivorous insects comprises the production of herbivore-induced plant volatiles (HIPVs). To unravel the precise oxylipin signaltransduction underlying the production of HIPVs in Arabidopsis thaliana and the resulting attraction of parasitoid wasps, we used a multidisciplinary approach that includes molecular genetics, metabolite analysis, and behavioral analysis. Mutant plants affected in the jasmonate pathway (18:0 and/or 16:0 -oxylipin routes; mutants dde2-2, fad5, opr 3 ) were studied to assess the effects of JA and its oxylipin intermediates 12-oxo-phytodienoate (OPDA) and dinor-OPDA (dnOPDA) on HIPV emission and parasitoid (Diadegma semiclausum) attraction. Interference with the production of the oxylipins JA and OPDA altered the emission of HIPVs, in particular terpenoids and the phenyl-
\end{abstract}

Electronic supplementary material The online version of this article (doi:10.1007/s10886-009-9696-3) contains supplementary material, which is available to authorized users.

Tjeerd A. L. Snoeren and Remco M. P. Van Poecke contributed equally to the manuscript.

T. A. L. Snoeren $(\bowtie) \cdot$ R. M. P. Van Poecke $\cdot$ M. Dicke Laboratory of Entomology, Wageningen University, PO Box 8031, 6700 EH Wageningen, The Netherlands e-mail: tjeerd.snoeren@wur.nl

Present Address:

R. M. P. Van Poecke

Keygene NV,

PO Box 216, 6700 AE Wageningen, The Netherlands propanoid methyl salicylate, which affected parasitoid attraction. Our data show that the herbivore-induced attraction of parasitoid wasps to Arabidopsis plants depends on HIPVs that are induced through the 18:0 oxylipin-derivative JA. Furthermore, our study shows that the 16:0-oxylipin route towards dnOPDA does not play a role in HIPV induction, and that the role of 18:0 derived oxylipinintermediates, such as OPDA, is either absent or limited.

Keywords Herbivory $\cdot$ Oxylipin $\cdot$ Herbivore-induced volatile - Metabolite analysis · Behavioral analysis . Pieris rapae

\section{Introduction}

Plants have evolved direct and indirect defenses that effectively combat attack by herbivorous insects (Kessler and Baldwin 2002). Direct defense mechanisms comprise the production and storage of metabolites that negatively influence herbivore performance (Wittstock and Gershenzon 2002). In contrast, indirect defense mechanisms encompass the production of metabolites that benefit the natural enemies of herbivores (Dicke et al. 1999; Wäckers et al. 2001; Halitschke et al. 2008; Kost and Heil 2008). Both defense mechanisms can be constitutively present or induced after herbivore feeding. Induced defenses allow plants to be more cost effective and also to diminish the risk that herbivores adapt to the defenses (Agrawal and Karban 1999; Pieterse and Dicke 2007; Steppuhn and Baldwin 2008). Plant responses to different herbivore species vary, as mediated by different feeding modes and defense elicitors in herbivore regurgitant or saliva (Walling 2000; Voelckel and Baldwin 2004; De Vos et al. 2005; Felton and Tumlinson 2008). 
Here, we addressed induced indirect plant defense at different levels of biological integration. An example of an induced indirect defense mechanism is the production of volatiles by plants in response to herbivory. These volatiles are used by parasitoids or predators to locate their herbivorous victims. Herbivore-induced plant volatiles (HIPVs) mainly comprise green leaf volatiles (GLVs), terpenoids, and phenolics (Dudareva et al. 2006). The composition of induced volatile blends can vary qualitatively or quantitatively (Dicke and Hilker 2003). With this variation, the plant can provide the natural enemies of herbivores with detectable and reliable information (Vet and Dicke 1992; Dicke 1999). The induced volatile production is orchestrated by at least three main signal-transduction pathways: the jasmonic acid (JA), salicylic acid (SA), and ethylene (ET) pathways (Dicke and Van Poecke 2002; Kessler and Baldwin 2002). These pathways can be differentially induced by different herbivore species (Heidel and Baldwin 2004; Schmidt et al. 2005; De Vos et al. 2005), leading to the emission of an herbivore-specific volatile blend (Vet and Dicke 1992; Ozawa et al. 2000; Walling 2000; Leitner et al. 2005).

Jasmonic acid (JA) is a member of a family of compounds collectively known as jasmonates or oxylipins, produced by the jasmonate pathway (Fig. 1). Leaf-feeding

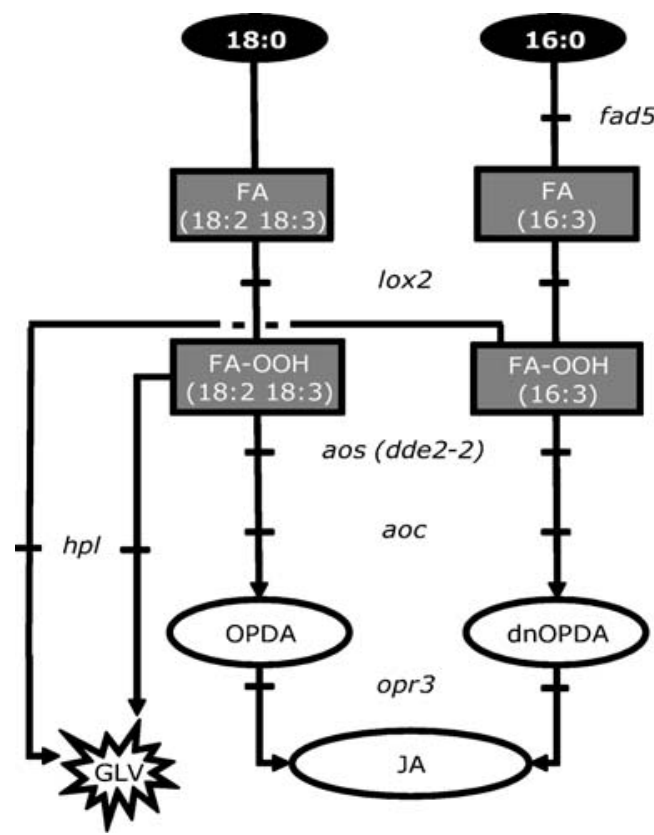

Fig. 1 Biosynthetic route of jasmonates in infested Arabidopsis thaliana leaves. FA Fatty Acid; 16:0 = hexadecanoic acid; 16:3=7Z, 10Z,13Z-hexadecatrienoic acid; $18: 0=$ octadecanoic acid; $18: 2=$ 9Z,12Z-octadecadienoic acid (linoleic acid); 18:3=9Z,12Z,15Zoctadecatrienoic acid (linolenic acid); $d n-O P D A$ dinor-oxophytodienoic acid; $O P D A$ oxo-phytodienoic acid; $J A$ jasmonic acid; $G L V S$ green leaf volatiles. The HPL pathway also utilizes 9- and 13hydroxides from hexadecanoic, linoleic and linolenic acid. Mutations affecting the biosynthesis are indicated in italics insects, such as the larvae of herbivorous Lepidoptera, especially induce the jasmonate pathway (Kessler and Baldwin 2002; De Vos et al. 2005). The production of jasmonates from linolenic acid (18:3) and linoleic acid $(18: 2)$ is initiated in the plastid and completed in the peroxisome and cytosol (Schaller et al. 2005). Lipases that release linolenic acid from membrane lipids, mainly originating from damaged cell walls, are thought to play an important role in regulating the response to herbivorederived cues (Farmer and Ryan 1992; Schaller et al. 2005). Linolenic acid and linoleic acid subsequently are converted by lipoxygenase (LOX) (Bell et al. 1995), allene-oxide synthase (AOS) (Laudert and Weiler 1998), and alleneoxide cyclase (AOC) (Ziegler et al. 1997) into 12oxophyto-dienoic acid (OPDA). A parallel cascade converts hexadecatrienoic acid (16:3) to dinor-oxophytodienoic acid (dnOPDA) (Weber et al. 1997). After the activity of 12 oxophytodienoic acid reductase (OPR) (Stintzi and Browse 2000) and three B-oxidation steps, the oxylipins OPDA and dnOPDA are metabolized to form JA (Schaller et al. 2005). Another branch, starting at 9- or 13-hydroperoxide formed by the lipoxygenase, leads to the production of GLVs through the action of hydroperoxide lyase (HPL) (Bate et al. 1998).

Thus, herbivory results in a change in the oxylipin signature in a plant (Vollenweider et al. 2000). However, it remains to be unraveled what the relative contribution of the different oxylipins is in the induction of indirect defense in terms of HIPV emission and the attraction of natural enemies of herbivores. Evidence for the involvement of jasmonates in herbivore-induced responses does not stem only from the induction of JA and its intermediates upon herbivory. Exogenous application of jasmonates mimics the effects of herbivory. For example, exogenous application of methyl jasmonate (MeJA) and caterpillar-feeding induce in Arabidopsis similar, but not identical, transcriptional responses (Reymond et al. 2004). De Vos et al. (2005) demonstrated that there is roughly a $50 \%$ overlap in gene induction in Arabidopsis after MeJA treatment and herbivory by caterpillars or thrips. Besides MeJA, other jasmonates also trigger transcriptional changes. Interestingly, exogenous treatment with OPDA, JA, or MeJA results in overlapping but not identical gene-expression profiles in Arabidopsis (Taki et al. 2005).

Second, jasmonates also influence the emission of volatiles by plants. The production of volatiles by JAtreated plants is quantitatively and qualitatively similar compared to induction by herbivory in Lima bean (Dicke et al. 1999; Ozawa et al. 2000). Application of OPDA has effects on secondary metabolite production similar to applying MeJA, in cell cultures of several plant species (Gundlach and Zenk 1998). However, exogenously applied OPDA but not JA induces diterpenoids in Lima bean plants (Koch et al. 1999). 
A third line of evidence for the involvement of jasmonates in herbivore-induced responses comes from mutant analyses. For example, Arabidopsis opr3 and aos mutants show different gene-expression profiles in response to JA, MeJA, OPDA, and mechanical damage compared to wild-type plants, thus indicating distinct signaling roles for dnOPDA, OPDA and JA (Stintzi et al. 2001; Taki et al. 2005). Indeed, opr3 mutants that lack JA still show oxylipin-dependent resistance to pathogens and herbivores, implying a role for jasmonates other than JA in plant defense (Stintzi et al. 2001).

Mutations in the oxylipin pathway also affect indirect defense. In Nicotiana attenuata for example, AOS-silenced plants (as-aos), display a reduced JA accumulation and terpenoid emission, and antisense- $h p l$ mutants release fewer GLVs (Halitschke et al. 2004). Similarly, two antisense- $h p l-$ mutants show an altered production of GLVs in Arabidopsis (Shiojiri et al. 2006a). These oxylipin-mediated effects on volatile emissions are accompanied by effects on interactions of plants with natural enemies of herbivores (Shiojiri et al. 2006b; Halitschke et al. 2008).

Thus, several lines of evidence demonstrate the involvement of jasmonic acid in herbivore-induced responses, including indirect defense, yet also suggest roles for other intermediates of the jasmonate pathway such as dnOPDA or OPDA. As predators and parasitoids are able to discriminate JA-induced from herbivore-induced volatiles (Dicke et al. 1999; Gols et al. 1999; Van Poecke and Dicke 2002), other signals besides JA are likely required for the induction of indirect defenses. Mutant plants altered in signaling pathways with a changed volatile emission represent some of the most powerful tools of testing the mechanisms that underlie HIPV production. The investigation of the effects of altered HIPV production in behavioral assays then is a valuable key to unravel the ecological relevance of these signaling pathways. Here, we followed a molecular ecological approach to study the involvement of several intermediates from the jasmonate pathway in the induction of plant volatiles by leaf-feeding herbivores. We used Arabidopsis thaliana to dissect the jasmonate pathway and analyze the effects on indirect defense after attack by leaf-feeding herbivores. In particular, we were interested in the contribution of the two sub-pathways that originate from galactolipids (16:0) or phospholipids (18:0) (Schaller et al. 2005), with special interest in dnOPDA, OPDA, and JA. For this, we selected mutants with altered production levels of dnOPDA, OPDA, and JA (Weber et al. 1997; Stintzi and Browse 2000; Stintzi et al. 2001; Von Malek et al. 2002). For caterpillar-infested mutants and their corresponding wild-type plants, the levels of dnOPDA, OPDA, and JA were quantified. Subsequently, HIPVs were collected, and volatile blend composition was quantitatively analyzed. Finally, we quantified caterpillar-feeding rate and conducted behavioral bioassays with parasitoid wasps to determine the effects of the observed differences in oxylipin profiles and HIPV blend composition after caterpillarfeeding on species interactions.

\section{Methods and Materials}

Plants and Insect Material Arabidopsis seeds (A. thaliana; genotypes Columbia (Col-0), Wassilewskija (WS), fad5, opr3, and dde2-2) were germinated on an autoclaved mixture of commercially available potting soil and $33 \%$ sand, and cultivated in a growth chamber at $21 \pm 2{ }^{\circ} \mathrm{C}, 50$ to $60 \%$ relative humidity $(\mathrm{RH})$, and L8:D16h photoperiod with 80 to $110 \mu \mathrm{mol} \mathrm{m} \mathrm{mec}^{-1} \mathrm{PPF}$. The selected mutant fad5 has a Col-0 background, and is incapable of biosynthesizing 7Z,10Z,13Z-hexadecatrienoic acid (16:3) (Weber et al. 1997); the mutant dde2-2 also has a Col-0 background and is defective in allene oxide-synthase (AOS) (Von Malek et al. 2002). The mutant opr3 has a WS background and lacks the most relevant isozyme of 12oxo-phytodienoate reductase (OPR) (Schaller et al. 2000; Stintzi and Browse 2000; Stintzi et al. 2001). Two-wk-old seedlings were transferred to plastic cups ( $5 \mathrm{~cm}$ diam) filled with the earlier described soil mixture. Plants were watered twice a week. When plants were full-grown, vegetative plants, i.e., 6-8 wk after sowing, they were used for experiments.

Herbivore-induced defense responses were initiated by caterpillars from Pieris rapae, the small cabbage white. Pieris rapae was reared on Brussels sprouts plants (Brassica oleracea var. gemmifera, cv Cyrus) in a climatized room (L16:D8h; $20 \pm 2^{\circ} \mathrm{C}$ and $70 \% \mathrm{RH}$ ).

The parasitoid wasp Diadegma semiclausum was reared on Plutella xylostella caterpillars feeding on Brussels sprouts in a climatized room (L16:D8h; $20 \pm 2{ }^{\circ} \mathrm{C}$ and $70 \%$ $\mathrm{RH})$. Emerging wasp species were provided ad libitum with water and honey for 2-5 d until experiments were conducted, and are referred to as 'naïve' wasps, as they had received no exposure to plant material, nor an oviposition experience.

Plant Treatments Defense responses were induced by herbivore feeding, or by spraying the plant with JA. Plants were infested by equally distributing 20 first-instar $P$. rapae larvae over the fully expanded leaves. Herbivore feeding was mimicked by spraying JA. Four plants were sprayed with $5 \mathrm{ml}$ of $1.0 \mathrm{mM}( \pm)-\mathrm{JA}$ (Sigma-Aldrich) aqueous solution. JA treatment was performed outside the climate room, for ca $15 \mathrm{~min}$, under comparable climatic conditions. After treatment, plants were placed back into the climate room. In all experiments, plants were treated $24 \mathrm{~h}$ before the experiments and kept in a climate room $\left(21 \pm 2^{\circ} \mathrm{C}\right.$, 50-60\% RH; L8:D16h photoperiod and 80 to $110 \mu \mathrm{mol} \mathrm{m}{ }^{-2} \mathrm{sec}^{-1}$ PPFD). 
Quantitative Analysis of Jasmonate Family Members The abundance of dnOPDA, OPDA, and JA, was determined for each of the used Arabidopsis genotypes. After $24 \mathrm{~h}, P$. rapae larvae were removed. All rosette leaves from infested or uninfested control plants were harvested, immediately weighed, and frozen in liquid nitrogen for storage. Extraction of the oxylipins was performed according to the protocol described by Weber et al. (1997). For quantifying the derivatized oxylipins, a gas chromatograph (Hewlett-Packard 5890) equipped with a $30 \mathrm{~m} \times 0.25 \mathrm{~mm}$ HP-MS column (Hewlett-Packard) coupled to a massspectrometer (model 5972, Hewlett-Packard) was used. Helium was used as carrier gas with a flow of $1 \mathrm{ml} \mathrm{min}{ }^{-1}$. The column temperature at the moment of injection was $100^{\circ} \mathrm{C}$. The temperature gradient was $100^{\circ} \mathrm{C}$ to $160^{\circ} \mathrm{C}$ at $20^{\circ} \mathrm{C} \mathrm{min}{ }^{-1}, 160^{\circ} \mathrm{C}$ to $238^{\circ} \mathrm{C}$ at $3^{\circ} \mathrm{C} \mathrm{min}^{-1}$, and $238^{\circ} \mathrm{C}$ to $300^{\circ} \mathrm{C}$ at $30^{\circ} \mathrm{C} \min ^{-1}$. Quantification was done by measuring selective ions $m / z=224$ for methyl jasmonate, $m / z=278$ for methyl dnOPDA, $m / z=238$ for methyl OPDA, $m / z=226$ for methyl dihydrojasmonate (Internal Standard used for methyl JA), and $m / z=240$ for methyl tetrahydroOPDA (Internal Standard used for methyl dnOPDA and methyl OPDA). Internal standards, methyl dihydrojasmonate and methyl tetrahydro-OPDA, were synthesized according to the protocol described by Weber et al. (1997).

Amounts of dnOPDA, OPDA, and JA were calculated per gram fresh weight. In cases where the oxylipin quantity was below the detection level for a certain sample, a value of 0.01 was assigned to these samples. Values were $\log 10$ transformed. The following fixed effects-model was used for each oxylipin to screen for differentiation per genotype: $\log 10\left(\mathrm{O}_{\mathrm{ij}}\right) \sim \mathrm{G}_{\mathrm{i}}+\mathrm{T}_{\mathrm{j}}+\mathrm{G}: \mathrm{T}_{\mathrm{ij}}+\varepsilon_{\mathrm{ij}}$, where $\mathrm{O}=$ oxylipin quantity per gram fresh weight; $\mathrm{G}=$ genotype; $\mathrm{T}=$ treatment; $\varepsilon=$ residual; $i=1, \ldots, 4$; and $j=1,2$. Subsequently, per oxylipin two-tailed $t$-tests among Genotype:Treatment combinations were performed to obtain $P$ values. For the 2004 data, the $t$-tests were followed by a Benjamini and Hochberg false discovery rate (BH-FDR) multiple comparison correction, to obtain the $q$ values (Benjamini and Hochberg 1995). All linear models were performed in the $\mathrm{R}$ environment ( $\mathrm{R}$ Development Core Team 2008) with $\mathrm{R}$ packages for linear mixed-effects models.

Headspace Collection and Volatile Analysis Dynamic headspace sampling was done for sets of 4 plants in a climate room $\left(20 \pm 2^{\circ} \mathrm{C}, 70 \% \mathrm{RH}\right.$; L8:D16h photoperiod and 90 to $110 \mu \mathrm{mol} \mathrm{m}{ }^{-2} \mathrm{sec}^{-1}$ PPFD). Twenty-four h before trapping, the pots were removed, soil with the roots were carefully wrapped in aluminum foil, and the 4 plants were placed together in 2.5-liter glass jar. Plants either were left uninfested or were infested with 20 first-instar (L1) $P$. rapae per plant. Just before trapping, jars were closed with a Viton-lined inert glass lid having an inlet and outlet. Air was sucked out with a vacuum pump at $100 \mathrm{ml} \mathrm{min}^{-1}$ with the incoming air purified through a steel cartridge filled with 200 mg Tenax-TA (20/35-mesh, Grace-Alltech, Deerfield, MI, USA). A same kind of cartridge was used to trap emitted plant volatiles at the outlet. After $3.5 \mathrm{~h}$ of trapping at continuous light, fresh weights of the 4 plants were measured. Headspace collections of uninfested and infested plants, for all the genotypes, were carried out in parallel on one experimental day.

Headspace samples were analyzed with a Thermo TraceGC Ultra $^{\mathrm{TM}}$ (Thermo Fisher Scientific, Waltham, MA, USA) connected to a Thermo TraceDSQ quadrupole mass spectrometer (Thermo Fisher Scientific, Waltham, MA, USA). Before desorption of the volatiles, the cartridges were dry-purged with helium at $30 \mathrm{ml} \mathrm{min}^{-1}$ for $20 \mathrm{~min}$ at ambient temperature to remove moisture. Samples were desorbed from the cartridges by using a thermal desorption system at $250^{\circ} \mathrm{C}$ for $3 \mathrm{~min}$ (Model Ultra Markes Llantrisant, UK) with a helium flow at $30 \mathrm{ml} \mathrm{min}{ }^{-1}$. Analytes were focused at $0^{\circ} \mathrm{C}$ on an electronically-cooled sorbent trap filled with Tenax and Carbograph (Unity ${ }^{\mathrm{TM}}$, Markes International LTD, Llantrisant, UK). Volatiles were transferred without split to the analytical column (Rtx 5MS, $30 \mathrm{~m} \times 0.25 \mathrm{~mm}$ i.d., $1.0 \mu \mathrm{m}$ film thickness, Restek, Bellefonte, PA, USA) by ballistic heating of the cold trap to $300^{\circ} \mathrm{C}$. The GC was held at an initial temperature of $40^{\circ} \mathrm{C}$ for $3.5 \mathrm{~min}$ followed by a linear thermal gradient of $10^{\circ} \mathrm{C} \mathrm{min}{ }^{-1}$ to $280^{\circ} \mathrm{C}$ and held for $2.5 \mathrm{~min}$ with a

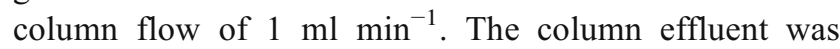
ionized by electron impact ionization at $70 \mathrm{eV}$. Mass spectra were acquired by scanning from 45 to $400 \mathrm{~m} / \mathrm{z}$ with a scan rate of 3 scans $\sec ^{-1}$.

Compounds were identified by using the deconvolution software (AMDIS version 2.64, NIST, USA) in combination with NIST 98 and Wiley 7th edition spectral libraries and by comparing their retention indices with those from literature (Adams 1995). Characteristic quantifier ions were selected for each compound of interest. Metalign software (PRIRikilt, Wageningen, The Netherlands) was used to align chromatograms of all samples and integrate peak areas for the signals of the quantifier ions. Peak areas were converted to peak area per gram fresh weight of leaf material.

Areas of quantifier ions per gram fresh weight were $\log 10$ transformed, and for each HIPV compound the following mixed model was used to screen for HIPV compound differentiation per genotype: $\log 10\left(\mathrm{~V}_{\mathrm{ijk}}\right) \sim \mathrm{G}_{\mathrm{i}}+\mathrm{T}_{\mathrm{j}}+\mathrm{G}$ : $T_{\mathrm{ij}}+\mathrm{R}_{\mathrm{k}}+\varepsilon_{\mathrm{ijk}}$, where $\mathrm{V}=$ area of quantifier ions per gram fresh weight; $\mathrm{G}=$ genotype; $\mathrm{T}=$ treatment; $\mathrm{R}=$ replicate; $\varepsilon=$ residual; $i=1, \ldots, 5 ; j=1,2$; and $k=1, \ldots, 5$. Both $\mathrm{G}$ and $\mathrm{T}$ were used as fixed effects and $\mathrm{R}$ as a random effect. Subsequently, two-tailed $t$-tests followed by a Benjamini and Hochberg false discovery rate (BH-FDR) multiple comparison correction were conducted per compound for the genotypes (Benjamini and Hochberg 1995). 
Similarities among plant genotypes based on volatile profiles were analyzed by using LEGG, a nonlinear dimensionality reduction method based on uncentered Pearson correlations among volatile profiles, which are used to generate 3-dimensional networks, for details see Van Poecke et al. (2007).

Behavioral Assays The effects of HIPV alterations as a result of mutations in the jasmonate pathway on behavioral responses were tested for the parasitoid species Diadegma semiclausum. Behavioral assays were carried out in a closed Y-tube olfactometer system as described in detail by Takabayashi and Dicke (1992). To investigate the behavioral responses of 3 to 7 -d-old mated D. semiclausum females, a modified Y-tube olfactometer was used (Bukovinszky et al. 2005). In short, filtered air was led through activated charcoal and split into two air streams (4 $1 \mathrm{~min}^{-1}$ ) that were led through 5-liter glass vessels containing the odor sources that consisted of 4 plants each. Plants were infested with 20 L1 P. rapae or sprayed with $1.0 \mathrm{mM}$ JA solution $24 \mathrm{~h}$ before starting the bioassay. Plants were kept overnight in a climate room $\left(21 \pm 2^{\circ} \mathrm{C}\right.$ and 50 to $60 \% \mathrm{RH}, \mathrm{L} 8: \mathrm{D} 16 \mathrm{~h}$ photoperiod and 80 to $\left.110 \mu \mathrm{mol} \mathrm{m}{ }^{-2} \mathrm{sec}^{-1} \mathrm{PPF}\right)$. The olfactometer was illuminated with 4 high-frequency fluorescent tubes (Philips $840,36 \mathrm{~W})$ from above at an intensity of $60 \pm 5 \mu \mathrm{mol}$ photons $\mathrm{m}^{-2} \mathrm{sec}^{-1}$. All experiments were conducted in a climatized room $\left(20 \pm 2^{\circ} \mathrm{C}\right)$.

Individual wasps were transferred into the Y-tube olfactometer, and their behavior was observed and scored as described in detail by Bukovinszky et al. (2005). Odor sources were interchanged to compensate for any unforeseen asymmetry in the set-up after every 5 wasps tested. Choices between odor sources were statistically analyzed by using a chi-square test, with the null-hypothesis that no preference existed.

The total area of consumed leaf-tissue was analyzed for the plants used in the bioassay. Therefore, after an experiment, all individual leaves of each rosette were taped on paper and scanned with a Hewlett-Packard scan jet 3570c. For quantification of the consumed leaf area, analysis was performed with KS400 version 3.0 software service pack 9 (Carl Zeiss Vision, Oberkochen, Germany). The consumed leaf area per genotype was statistically compared by using ANOVA (SPSS 15.0, Chicago, IL, USA).

\section{Results}

Quantitative Analysis of Jasmonate Family Members We quantified the levels of dnOPDA, OPDA, and JA in leaftissue from the mutants $d d e 2-2$, opr 3 , and fad5, after $24 \mathrm{~h}$ of herbivory by Pieris rapae, and from uninfested plants
(Fig. 2, Supplementary Table 1). As the dde2-2 and fad5 mutants have a Col-0 background, while the opr 3 mutant has a WS background, both wild-type accessions were included as well.

Analyses of Col-0 were performed both in 2004 and 2005. Even though plants were grown in a controlled environment, the levels of oxylipins varied between the years (Fig. 2). Therefore, the 2004 dataset was analyzed separately from the 2005 dataset. Constitutive levels of OPDA and JA are

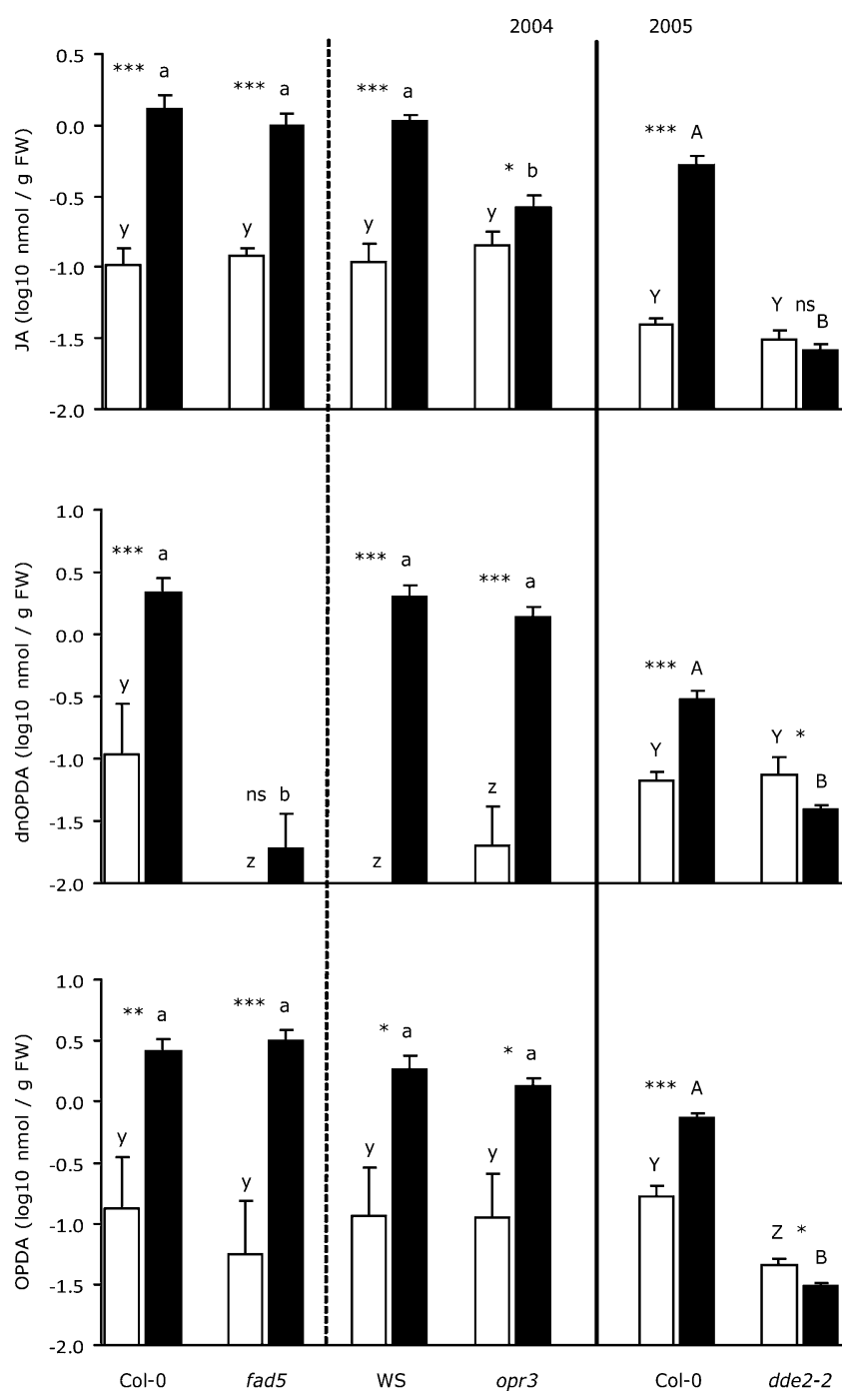

Fig. 2 Oxylipin amounts in Arabidopsis thaliana after $24 \mathrm{~h}$ of Pieris rapae feeding. Extracted oxylipin concentration for P. rapae-infested (black bar) or uninfested (white bar) leaves. Mean oxylipin values + SE are shown $(N=4$, see also Supplementary Table 1). Dashed line separates wild-type accession backgrounds for 2004. Asterisks indicate significant differences between infested and uninfested plants within a genotype $\left({ }^{*} P<0.05 ; * * P<0.01 ; * * * P<0.001 ; n s\right.$ not significantly different). Bars for infested leaves marked with the same letter are not significantly different (data from years 2004 and 2005 were analyzed independently, for the 2004 data we used lower case letters and for data from 2005 capitals) $(2004: q$ value $>0.05 ; 2005$ : $P$ value $>0.05$ ). dnOPDA amounts in undamaged fad5 and WS plants were below detection limit 
similar in both wild-type accessions, whereas constitutive dnOPDA levels are lower in WS compared to Col-0 (Fig. 2). Herbivory by $P$. rapae induced all three oxylipins to similar levels in Col-0 and WS plants (Fig. 2).

Mutations in oxylipin biosynthetic genes clearly affected the oxylipin signatures. In $d d e 2-2$ plants, induction of dnOPDA, OPDA, or JA by herbivory was completely abolished. In fact, a decrease in dnOPDA and OPDA levels was observed in dde2-2 plants in response to herbivory. Moreover, constitutive OPDA levels were lower in the dde2-2 mutant.

A mutation in FAD5 resulted in constitutively lower dnOPDA levels that could not be induced by herbivory. However, this mutation did not affect either constitutive or herbivore-induced levels of OPDA or JA.

A mutation in $O P R 3$ hampered the induction of JA after herbivory: herbivory resulted in significantly lower JA levels compared to the WS wild-type. Constitutive levels of all three oxylipins were unaffected by $O P R 3$ mutation.

In short, the oxylipin mutations have the expected effects on oxylipin production: the fad5 mutant only affects dnOPDA levels, opr 3 only affects JA levels, and dde2-2 affects the levels of dnOPDA, OPDA, and JA. Thus, these three mutants allow us to dissect the roles of dnOPDA, OPDA, and JA levels in indirect defense.

Volatile Analysis To assess the effects of altered oxylipin signatures on HIPVs, we measured the volatile emissions in uninfested and P. rapae-infested Col-0, WS, dde2-2, fad5, and opr 3 plants (Supplementary Table 2). Compounds for which an influence on discriminative behavior by carnivorous arthropods was known were selected and presented in Fig. 3 (De Boer et al. 2004; Dicke et al. 1990b; Shimoda et al. 2005). HIPV production in wild-type Col-0 and WS showed both similarities and differences. For example, $P$. rapae feeding induced the emission of methyl salicylate (MeSA), the sesquiterpene $(E, E)-\alpha$-farnesene, and the homoterpene $(E, E)$ 4,8,12-trimethyltrideca-1,3,7,11tetraene (TMTT) to similar levels in both accessions. However, Col-0, but not WS, showed the induction of the monoterpene linalool, whereas WS, but not Col-0, showed induction of the GLVs (Z)-3-hexenal and (Z)-3-hexen-1-ol acetate, and of the monoterpenes $\beta$-myrcene and both stereoisomers of $\beta$-ocimene.

A mutation in FAD5 did not result in an altered HIPV emission for the analyzed compounds, thus indicating that dnOPDA does not play a role in HIPV induction. In contrast, a mutation in DDE2-2 abolished the induction of all analyzed HIPVs, except for 1-nonanol. A mutation in $O P R 3$ blocked induction of all analyzed HIPVs, except for the GLVs.

To compare total volatile blends instead of single compounds among accessions, the variation in volatile
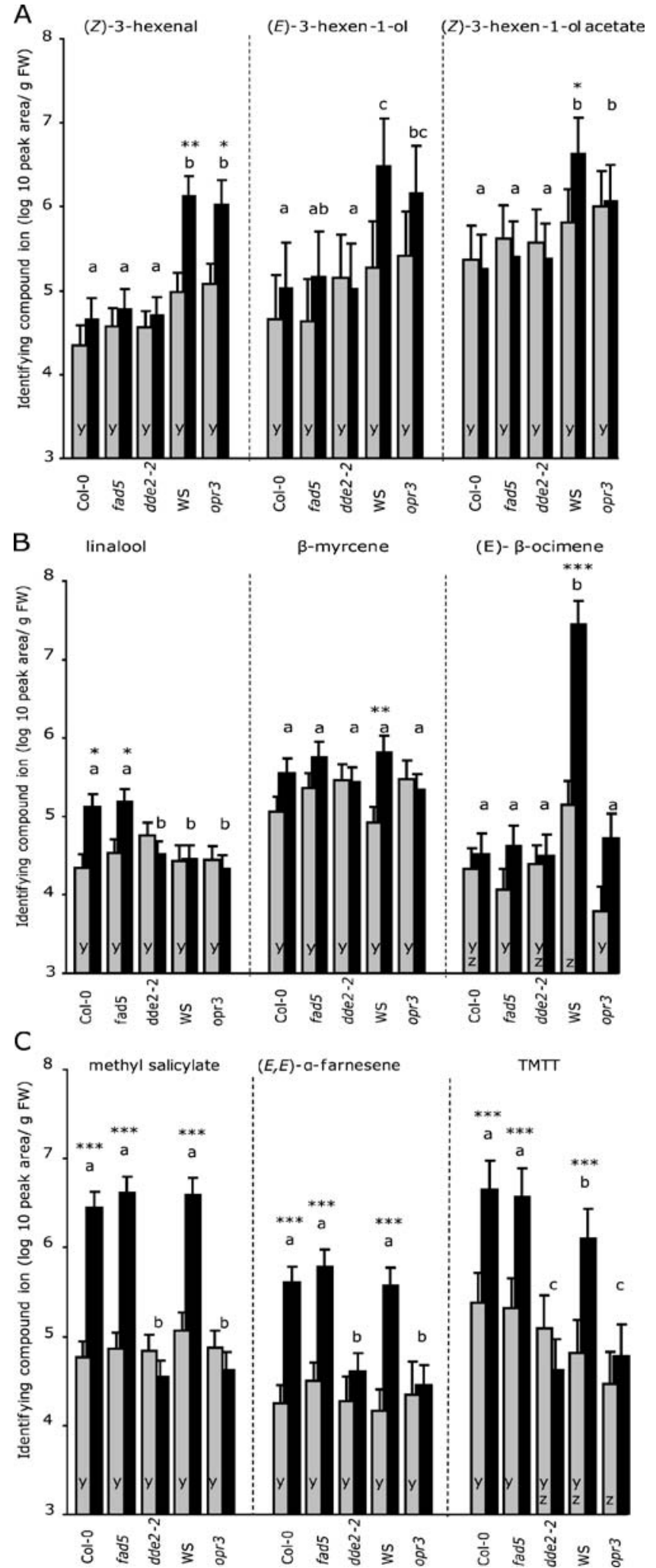

profiles among genotypes was explored further by using the algorithm locally linear embedding graph generator (LEGG). LEGG analysis resulted in a 2D network of genotypes, generated by using a non-linear dimensionality 
Fig. 3 Characteristic Arabidopsis thaliana HIPV-compounds per genotype treatment. a: GLVs; b: monoterpenes c: methyl salicylate (MeSA), $(E, E)-\alpha$-farnesene, and (E,E) 4,8,12-trimethyltrideca-1,3,7,11tetraene (TMTT). Given is the mean $+\mathrm{SE}$ for compound-representative ions $(N=4-5$ replicates per treatment, see also Supplementary Table 2). Asterisks indicate significant differences between uninfested (grey bars) and Pieris rapae-infested (black bars) leaves within a genotype $\left({ }^{*} P<\right.$ $0.05 ; * * P<0.01 ; * * * P<0.001)$. Bars that are marked with the same letter are not significantly different, $(\mathrm{y}-\mathrm{z}=$ uninfested plants, $\mathrm{a}-\mathrm{c}=$ infested plants), $q$ value $>0.05$. Dashed lines divide separately analyzed compounds

reduction method (Fig. 4) (for LEGG details see Van Poecke et al. (2007) and the legend of Fig. 4). This demonstrated a strong relationship between volatile profiles emitted by uninfested plants of the various genotypes plus caterpillar-infested plants of the dde2-2 and opr3 mutants. In contrast, HIPVs from caterpillar-infested Col-0, fad5, and WS showed a much weaker correlation with the volatile blend from uninfested genotypes. LEGG analysis also showed that the HIPVs from Col-0 and WS show similarities.

Thus, headspace analysis for the mutants $f a d 5$, dde2-2, opr 3 , and their respective wild-types pointed out that only hampered JA levels (i.e., the common denominator in dde22 and opr 3 plants) resulted in an altered production of HIPVs.

Behavioral Assays To investigate whether the changes in HIPV profiles due to the mutations in the jasmonate pathway affect indirect defense, we investigated the behavioral responses of a parasitoid wasp species towards HIPVs originating from $d d e 2-2, o p r 3$, fad5, and their wildtypes in a Y-tube olfactometer (Fig. 5). Diadegma semiclausum females preferred volatiles of infested plants over uninfested plants for all mutants or wild-types $(P<0.001)$. However, HIPVs from caterpillar-infested wild-type plants were significantly more attractive than HIPVs from caterpillar-infested mutants dde2-2 or opr3 (for both mutants $P<0.05$ ). The wasps did not discriminate between volatiles of herbivore-infested fad5 plants and infested wild-type plants.

A possible explanation for the observed differences in HIPV emission and parasitoid attraction among mutants and wild-type plants is that the mutation altered the feeding behavior of the herbivore. A reduced attraction to infested mutant plants might have resulted from a reduced feeding rate on the mutant plants. However, this is not supported by our data on feeding rates: the amount of leaf area consumed did not differ when the caterpillars were feeding on plants of opr 3, fad5, or their wild-types WS and Col-0 (308 \pm 21.6 , $285 \pm 15.8,284 \pm 18.1,295 \pm 17.7$ mean $\mathrm{mm}^{2} \pm \mathrm{SE} /$ plant, respectively; $P>0.05$ ). Moreover, the caterpillars even consumed more leaf material of $d d e 2-2$ plants compared to wildtype Col-0 $\left(211 \pm 12.4\right.$ and $169 \pm 10.8$ mean $\mathrm{mm}^{2} \pm \mathrm{SE} /$ plant,

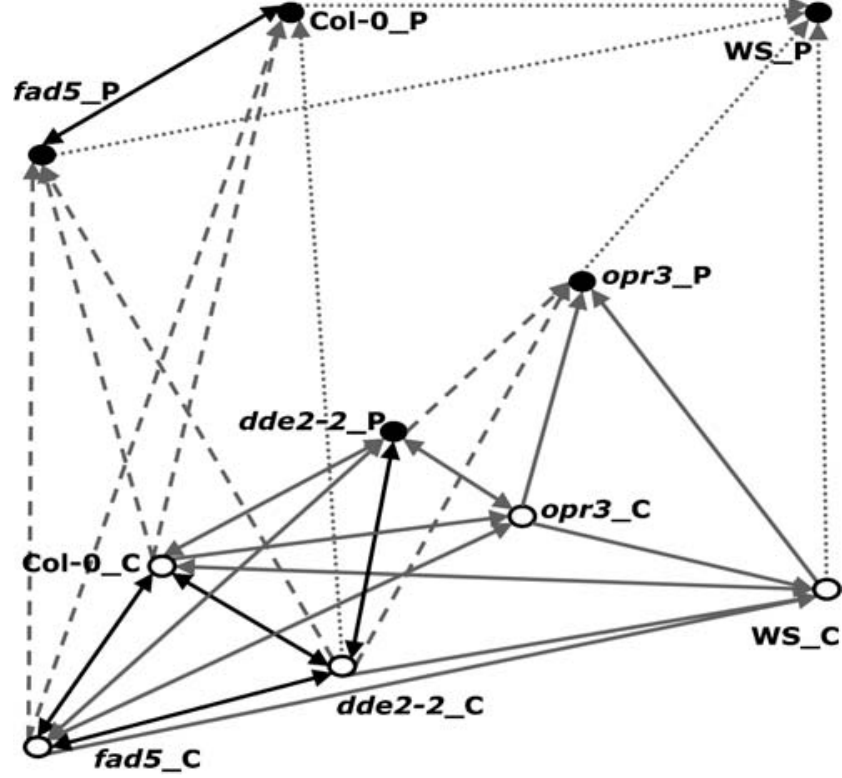

Fig. 4 Visualization of the relationships among HIPV blends of different Arabidopsis thaliana genotype-treatment combinations. To assess the similarities among volatile blends from different genotypetreatment combinations, LEGG analysis was applied to the volatile blends of the Pieris rapae-infested (_P ; black nodes) and uninfested ( $C$; white nodes) plants of different genotypes. Similarities were calculated as uncentered Pearson correlation coefficients, based on the quantitative information on 22 volatile compounds for each genotype treatment combination (1-pentanol; $(E)$-2-penten-1-ol; hexanal; $(Z)$-3hexenal; $(E)$-3-hexen-1-ol; 1-hexanol; heptanal; $\alpha$-pinene; 1-octen-3ol; 6-methyl-5-hepten-2-one; $\beta$-myrcene; $(Z)$-3-hexen-1-ol acetate; (Z)- $\beta$-ocimene; $(E)$ - $\beta$-ocimene; $(E)$-2-nonen-1-ol; linalool; $(E)-4,8$ dimethyl-1,3,7-nonatriene; 1-nonanol; methyl salicylate; undecanal; $(E, E)$ - $\alpha$-farnesene; $(E, E)$ 4,8,12-trimethyl-1,3,7,11-tridecatetraene). The similarities were visualized using non-linear dimensionality reduction. Similarities among the profiles that were determined by LEGG are depicted as directed links. Similarities can be compared based on the presence, absence or strength of the directed links. The strength of the similarities is indicated by shading and line-type. Arrow lengths do not illustrate strength of connections. Thus, solid black arrows connect blends from genotype-treatment combinations that are highly similar $\left(0.984<r^{2}<0.990\right)$. This similarity decreases from solid grey arrows $\left(0.974<r^{2}<0.984\right)$; dashed grey arrows $(0.964$ $\left.<r^{2}<0.974\right)$; to dotted grey arrows $\left(0.951<r^{2}<0.964\right)$. Blends from genotype-treatment combinations that are not connected are relatively dissimilar. A genotype-treatment combination at the base of an arrow represents a near neighbor of the genotype-treatment combination directed at. Arrows that point both ways represent genotypestreatment combinations that are both near neighbors of each other. For example, the volatile blend from P. rapae-infested dde2-2 mutants is highly similar to the blend from uninfested dde2-2 and other uninfested genotypes (nearest neighbours both ways, with relatively strong connections), whereas the blend of $P$. rapae infested Col-0 is quite dissimilar from uninfested Col-0 (uninfested Col-0 is a nearest neighbour of infested Col-0, but not the other way around, and the connection is relatively weak). In short, LEGG provides a relatively easy visual interpretation of similarities among volatile blends based on all 22 components 


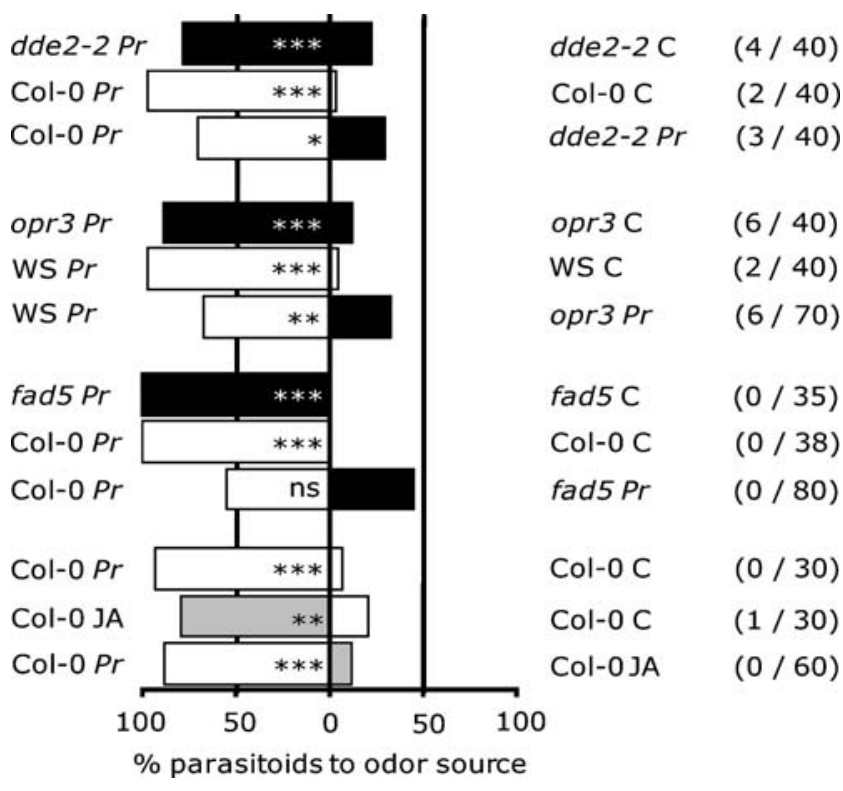

Fig. 5 Preference of naïve Diadegma semiclausum females to volatiles of differently treated Arabidopsis thaliana, as assessed in the Y-tube olfactometer. Plants were infested with Pieris rapae $(\mathrm{Pr})$ or treated with JA $24 \mathrm{~h}$ before (JA) or were left untreated (C). Data represent total number of parasitoids that chose for any of the two odor sources as determined in 3-4 replicate experiments, each on a different day with new odor sources. Asterisks indicate a significant difference within a choice test: $* P<0.05 ; * * P<0.01 ; * * * P<0.001 ; n s$ not significantly different $\left(\chi^{2}\right.$-test $)$. The number of wasps that did not make a choice and the total number of tested parasitoids is given in parenthesis

respectively; $P<0.05)$. Thus, the mutations do not result in reduced feeding and, thus, altered caterpillar-feeding rates cannot explain the observed changes in headspace composition or parasitoid attraction.

We verified the importance of JA signaling in indirect defense by assessing the attractiveness of volatiles induced in the plants by the application of $1 \mathrm{mM} \mathrm{JA}$. Exogenous JA treatment of Col-0 plants made them more attractive than non-treated Col-0 control plants (Fig. 5, $P<0.01$ ). However, when these JA-sprayed plants were tested against $P$. rapaeinduced Col-0, the wasps preferred the latter $(P<0.001)$.

\section{Discussion}

The jasmonate pathway plays a pivotal role in the induced plant defense response against many arthropod herbivores, including indirect defense responses. However, the role of the different (intermediate) products of this pathway in these defense responses is unclear. Even though it was shown recently that JA needs to be conjugated to an amino acid for functionality, and that OPDA does not serve as a substrate for the conjugating enzyme, there are several reports that indicate a distinct role of OPDA in direct and indirect defenses, possibly through its electrophile properties (Ribot et al. 2008). Therefore, we set out to unravel the involvement of the jasmonate pathway products dnOPDA, OPDA, and JA in caterpillar-induced indirect defense by using a molecular ecological approach. We selected Arabidopsis thaliana mutants, fad5, opr3, and dde2-2, for which we quantified oxylipin levels, HIPV emission, and ultimately assessed parasitoid attractiveness towards HIPVs.

Based on previous work, summarized in Fig. 1, we expected the fad5 mutant to show reduced levels of $16: 3$ derived compounds; the dde2-2 mutant to show reduced levels of both 16:3 and 18:3 derived compounds; and opr3 mutants to show reduced levels of JA. Our data confirmed these expectations: after herbivory fad5 showed no induction of dnOPDA, opr 3 was hampered in the induction of $\mathrm{JA}$, and dde2-2 showed no induction of dnOPDA, OPDA, and JA (Fig. 2). Additionally, these data also demonstrated that 1) the 16:3 pathway does not contribute to constitutive or herbivory-induced levels of JA, and 2) that herbivoryinduced levels of dnOPDA and OPDA do not depend on JA accumulation through a feedback loop.

Mutation of the FAD5 gene did not result in an altered emission of HIPVs, showing that dnOPDA does not play a role in HIPV-mediated indirect defense. On the other hand, mutations in DDE2-2 or OPR3 did show clear effects on HIPV production, resulting in reduced induction of many volatiles, especially the terpenoids, thus indicating roles of OPDA and/or JA in HIPV-indirect defense. As dde2-2 (which lacks both OPDA and JA) or opr3 (which lacks only JA) show very similar HIPV production, we conclude that JA, and not OPDA, is the most important oxylipin in HIPV production. A minor role for OPDA cannot be excluded, as opr 3 plants, showing moderate JA induction, still showed induction of a few volatile compounds, such as GLVs, while dde2-2 plants, lacking OPDA and JA induction, did not. However, it is likely that this difference between opr 3 and $d d e 2-2$ is caused by differences in the genetic background: none of the three genotypes with a Col-0 background (Col-0, fad5, and dde2-2) showed induction of GLVs, whereas WS did. The lower GLV-levels produced by Col-0, fad5, and $d d e 2-2$ plants are most likely caused by a dysfunctional HPL1 enzyme in the Col-0 background (Duan et al. 2005). In any case, the role of OPDA or earlier intermediates is minor, as LEGG analyses show that the composition of the overall volatile blend of opr 3 and dde2-2 plants is similar (Fig. 4). Moreover, the blends of infested dde2-2 and opr 3 plants were similar to the blends of uninfested controls, while they were different from the blends of infested wild-type plants (Fig. 4).

Parasitoid behavioral assays reflected the results obtained by headspace analyses: mutation of FAD5 did not affect parasitoid behavior, whereas infested plants of both opr3 and dde2-2 showed reduced parasitoid attraction 
compared to wild-type plants (Fig. 5). Some compounds were still induced in the mutants dde2-2 and opr3: 1-nonanol and (Z)-3-hexenal, respectively (Supplementary Table 2, Fig. 3). These compounds might explain the attractiveness of infested mutants dde2-2 and opr3 over uninfested controls. These results indicate that other signals besides JA or its intermediates are involved in HIPV production. This is illustrated also by the observation that JA-treated Col-0 plants were less attractive to the wasps than caterpillar-infested Col-0 (Fig. 5; see also Van Poecke and Dicke (2002)). Similar results also have been obtained for other plant-herbivore-carnivore systems (Bruinsma et al. 2009; Dicke et al. 1999).

The combination of biochemical and behavioral analyses of Arabidopsis wild-type and mutant plants not only gives insight into which plant hormones influence indirect defense, but also through which volatile compounds these hormones exert their effects. Inhibition of JA production influenced mono-, sesqui-, and homoterpene volatile emissions as well as the emission of the shikimate pathway-derived methyl salicylate (MeSA). Even though exogenous JA is known to induce GLV production in Arabidopsis (Van Poecke 2002), we did not find any effect of the lack of JA on $P$. rapae-induced GLV emissions in Arabidopsis.

The importance of monoterpenes in plant-insect interactions is well studied (Dicke et al. 1990a; Rose et al. 1998; Shimoda et al. 2005; Mumm et al. 2008; Opitz et al. 2008). Even though Col-0 and WS differed in the identity of monoterpenes induced by $P$ rapae feeding, with Col-0 showing mainly induction of linalool and WS showing mainly induction of $(Z)$ - and $(E)$ - $\beta$-ocimene, lack of JA had a similar inhibitory effect on monoterpene induction in both accessions. Lack of JA also affected emission rates of the sesquiterpene $(E, E)$ - $\alpha$-farnesene and the diterpenederivative $(E, E)$ 4,8,12-trimethyltrideca-1,3,7,11-tetraene (TMTT), demonstrating that JA influences not only the plastid-localized mono- and diterpene production, but also the sesquiterpene production located in the cytosol. Both $(E, E)-\alpha$-farnesene and TMTT are known to influence plantinsect interactions (Van Poecke 2002; De Boer et al. 2004; Ibrahim et al. 2005).

Besides terpenoids, the induction of MeSA also was impaired in JA-lacking mutants. This indicates that hampering the JA pathway consequently impedes some step in the biosynthesis of MeSA. Previous studies using Arabidopsis demonstrated that blocking the SA pathway affects the emission of both MeSA and TMTT and results in decreased parasitoid attraction (Van Poecke and Dicke 2002; Van Poecke 2002). Thus, both JA and SA are required for herbivore-induced emissions of MeSA and TMTT. Such synergism also has been recorded for spider-mite induced volatile emissions of tomato plants (Ament et al. 2004).
In summary, the present study has shown the value of using a molecular ecological approach for elucidating the relative importance of jasmonic acid and its intermediates in indirect defense. By dissecting the jasmonate pathway through the use of selected mutants, we have gained a better understanding of the ecological role of oxylipins in HIPV-mediated indirect plant defense. Even though the importance of the octadecanoid pathway in indirect defense had been demonstrated previously (Thaler et al. 2002; Halitschke et al. 2004; Kessler et al. 2004; Shiojiri et al. 2006a, b), the relative contribution of the different oxylipin compounds to indirect defense had remained unclear. Here, we demonstrated that in Arabidopsis the 16:0 branch of the oxylipin pathway does not play a role in HIPV-mediated indirect defense. Moreover, we demonstrated that from the 18:0 pathway, jasmonic acid is the main actor in HIPV-mediated indirect defense, with limited or no contribution from pathway intermediates such as OPDA. The data strengthen the value of using a molecular ecological approach in advancing our understanding of multi-trophic plant-insect interactions.

Acknowledgements The authors thank Leo Koopman, Frans van Aggelen, and André Gidding for culturing the insects, Ted farmer for training RMPvP to execute oxylipin signature analysis, Philippe Reymond for providing us with Arabidopsis seeds of mutants fad5 and opr3, Beat Keller for supplying us with seeds of Arabidopsis mutant dde2-2, Roland Mumm for critically reading an earlier version of the manuscript, Iris Kappers for help with volatile analysis, and Roland Mumm and Iris Kappers for fruitful discussion, Harro Bouwmeester for providing facilities at Plant Research International, and the Netherlands Organization for Scientific Research (NWO/ ALW) for a VICI grant (865.03.002; T.A.L.S. and M.D.).

Open Access This article is distributed under the terms of the Creative Commons Attribution Noncommercial License which permits any noncommercial use, distribution, and reproduction in any medium, provided the original author(s) and source are credited.

\section{References}

ADAMS, R. P. 1995. Identification of Essential Oil Components by Gas Chromatography/Mass Spectrometry. Allured Publishing Corporation, Illinois.

AgraWAL, A. A. and Karban, R. 1999. Why induced defenses may be favored over constitutive strategies in plants, pp. 45-61, in R. Tollrian and C. D. Harvell (eds.). The Ecology and Evolution of Inducible Defenses. Princeton University Press, Princeton, N.J.

Ament, K., Kant, M. R., Sabelis, M. W., Haring, M. A., and SCHUURINK, R. C. 2004. Jasmonic acid is a key regulator of spider mite-induced volatile terpenoid and methyl salicylate emission in tomato. Plant Physiol. 135:2025-2037.

Bate, N. J., Sivasankar, S., Moxon, C., Riley, J. M. C., Thompson, J. E., and Rothstein, S. J. 1998. Molecular characterization of an Arabidopsis gene encoding hydroperoxide lyase, a cytochrome P-450 that is wound inducible. Plant Physiol. 117:1393-1400.

Bell, E., Creelman, R. A., and Mullet, J. E. 1995. A chloroplast lipoxygenase is required for wound-induced jasmonic acid 
accumulation in Arabidopsis. Proc. Natl. Acad. Sci. USA 92:8675-8679.

Benjamini, Y. and HochBerg, Y. 1995. Controlling the false discovery rate-A practical and powerful approach to multiple testing. J. Roy. Stat. Soc. Ser. B. (Stat. Method.) 57:289-300.

Bruinsma, M., Posthumus, M. A., Mumm, R., Mueller, M. J., VAN LOON, J. J. A., and DiCKE, M. 2009. Jasmonic acid-induced volatiles of Brassica oleracea attract parasitoids: effects of time and dose, and comparison with induction by herbivores. J. Exp. Bot. 60:2575-2587.

Bukovinszky, T., Gols, R., Posthumus, M. A., Vet, L. E. M., and VAN LENTEREN, J. C. 2005. Variation in plant volatiles and attraction of the parasitoid Diadegma semiclausum (Hellen). $J$. Chem. Ecol. 31:461-480.

De Boer, J. G., Posthumus, M. A., and Dicke, M. 2004. Identification of volatiles that are used in discrimination between plants infested with prey or nonprey herbivores by a predatory mite. J. Chem. Ecol. 30:2215-2230.

De Vos, M., Van Oosten, V. R., Van Poecke, R. M. P., Van Pelt, J. A., Pozo, M. J., Mueller, M. J., Buchala, A. J., MetrauX, J. P., VAN LOON, L. C., DiCKE, M. et al. 2005. Signal signature and transcriptome changes of Arabidopsis during pathogen and insect attack. Mol. Plant-Microbe Interact. 18:923-937.

DiCKE, M. 1999. Are herbivore-induced plant volatiles reliable indicators of herbivore identity to foraging carnivorous arthropods? Entomol. Exp. Appl. 91:131-142.

DiCKE, M. and HILKER, M. 2003. Induced plant defences: from molecular biology to evolutionary ecology. Basic Appl. Ecol. 4:3-14.

Dicke, M. and VAN PoECKE, R. M. P. 2002. Signaling in plant-insect interactions: signal transduction in direct and indirect plant defence, pp. 289-316, in D. Scheel and C. Wasternack (eds.). Plant Signal Transduction. Oxford University Press, Oxford.

Dicke, M., MaAs Van Der, K. J., Takabayashi, J., and Vet, L. E. M. 1990a. Learning affects response to volatile allelochemicals by predatory mites. Proc. Exp. Appl. Entomol. 1:31-36.

Dicke, M., Van Beek, T. A., Posthumus, M. A., Ben Dom, N., Van BokHoven, H., and DE GroOT, A. E. 1990b. Isolation and identification of volatile kairomone that affects acarine predatorprey interactions. Involvement of host plant in its production. $J$. Chem. Ecol. 16:381-396.

Dicke, M., Gols, R., Ludeking, D., and Posthumus, M. A. 1999. Jasmonic acid and herbivory differentially induce carnivoreattracting plant volatiles in lima bean plants. J. Chem. Ecol. 25:1907-1922.

Duan, H., Huang, M. Y., Palacio, K., and Schuler, M. A. 2005. Variations in CYP74B2 (hydroperoxide lyase) gene expression differentially affect hexenal signaling in the Columbia and Landsberg erecta ecotypes of Arabidopsis. Plant Physiol. 139:1529-1544.

Dudareva, N., Negre, F., Nagegowda, D. A., and Orlova, I. 2006. Plant volatiles: Recent advances and future perspectives. Crit. Rev. Plant Sci. 25:417-440.

FARMER, E. E. and RYAN, C. A. 1992. Octadecanoid precursors of jasmonic acid activate the synthesis of wound-inducible proteinase inhibitors. Plant Cell 4:129-134.

Felton, G. W. and Tumlinson, J. H. 2008. Plant-insect dialogs: complex interactions at the plant-insect interface. Curr. Opin. Plant Biol. 11:457-463.

Gols, R., Posthumus, M. A., and Dicke, M. 1999. Jasmonic acid induces the production of gerbera volatiles that attract the biological control agent Phytoseiulus persimilis. Entomol. Exp. Appl. 93:77-86.

GundLACH, H. and ZENK, M. H. 1998. Biological activity and biosynthesis of pentacyclic oxylipins: The linoleic acid pathway. Phytochemistry 47:527-537.
Halitschke, R., Ziegler, J., Keinanen, M., and Baldwin, I. T. 2004. Silencing of hydroperoxide lyase and allene oxide synthase reveals substrate and defense signaling crosstalk in Nicotiana attenuata. Plant J. 40:35-46.

Halitschke, R., Stenberg, J. A., Kessler, D., Kessler, A., and BALDWIN, I. T. 2008. Shared signals - 'alarm calls' from plants increase apparency to herbivores and their enemies in nature. Ecol. Lett. 11:24-34.

HeIDEL, A. J. and BALDWIN, I. T. 2004. Microarray analysis of salicylic acid- and jasmonic acid-signalling in responses of Nicotiana attenuata to attack by insects from multiple feeding guilds. Plant Cell Environ. 27:1362-1373.

IbrahiM, M. A., NisSINEN, A., and HolopainEN, J. K. 2005. Response of Plutella xylostella and its parasitoid Cotesia plutellae to volatile compounds. J. Chem. Ecol. 31:1969-1984.

Kessler, A. and BALDwIN, I. T. 2002. Plant responses to insect herbivory: The emerging molecular analysis. Annu. Rev. Plant Biol. 53:299-328.

Kessler, A., HalitschKe, R., and Baldwin, I. T. 2004. Silencing the jasmonate cascade: Induced plant defenses and insect populations. Science 305:665-668.

Koch, T., Krumm, T., Jung, V., Engelberth, J., and Boland, W. 1999. Differential induction of plant volatile biosynthesis in the lima bean by early and late intermediates of the octadecanoidsignaling pathway. Plant Physiol. 121:153-162.

Kost, C. and HeIL, M. 2008. The defensive role of volatile emission and extrafloral nectar secretion for lima bean in nature. J. Chem. Ecol. 34:2-13.

LAUDERT, D. and WeILER, E. W. 1998. Allene oxide synthase: a major control point in Arabidopsis thaliana octadecanoid signalling. Plant J. 15(5):675-684.

Leitner, M., Boland, W., and Mithofer, A. 2005. Direct and indirect defences induced by piercing-sucking and chewing herbivores in Medicago truncatula. New Phytol. 167:597-606.

Mumm, R., Posthumus, M. A., and Dicke, M. 2008. Significance of terpenoids in induced indirect plant defence against herbivorous arthropods. Plant Cell Environ. 31:575-585.

Opitz, S., Kunert, G., and Gershenzon, J. 2008. Increased terpenoid accumulation in cotton (Gossypium hirsutum) foliage is a general wound response. J. Chem. Ecol. 34:508-522.

OzaWA, R., Arimura, G., TAKabayashi, J., Shimoda, T., and NISHIOKA, T. 2000. Involvement of jasmonate- and salicylaterelated signaling pathways for the production of specific herbivore-induced volatiles in plants. Plant Cell Physiol. 41:391-398

Pieterse, C. M. J. and DiCKE, M. 2007. Plant interactions with microbes and insects: from molecular mechanisms to ecology. Trends Plant Sci. 12:564-569.

R Development Core TEAm (2008). R: A language and environment for statistical computing. R Foundation for Statistical Computing, Vienna, Austria. ISBN 3-900051-07-0, URL http:// www.R-project.org.

Reymond, P., Bodenhausen, N., VAn Poecke, R. M. P., KrishnaMURTHY, V., DICKE, M., and FARMER, E. E. 2004. A conserved transcript pattern in response to a specialist and a generalist herbivore. Plant Cell 16:3132-3147.

Ribot, C., Zimmerli, C., FArmer, E. E., Reymond, P., and Poirier, Y. 2008. Induction of the Arabidopsis PHO1;H10 gene by 12oxo-phytodienoic acid but not jasmonic acid via a Coronatine Insensitive1-dependent pathway. Plant Physiol. 147:696706.

Rose, U. S. R., Lewis, W. J., and Tumlinson, J. H. 1998. Specificity of systemically released cotton volatiles as attractants for specialist and generalist parasitic wasps. J. Chem. Ecol. 24:303-319. 
Schaller, F., Biesgen, C., Mussig, C., Altmann, T., and Weiler, E. W. 2000. 12-oxophytodienoate reductase 3 (OPR3) is the isoenzyme involved in jasmonate biosynthesis. Planta 210:979-984.

Schaller, F., Schaller, A., and Stintzi, A. 2005. Biosynthesis and metabolism of jasmonates. J. Plant Growth Regul. 23:179-199.

Schmidt, D. D., Voelckel, C., Hartl, M., Schmidt, S., and BALDWIN, I. T. 2005. Specificity in ecological interactions. Attack from the same lepidopteran herbivore results in speciesspecific transcriptional responses in two solanaceous host plants. Plant Physiol. 138:1763-1773.

Shimoda, T., OzaWA, R., SANO, K., YANO, E., and TAKabayashi, J. 2005. The involvement of volatile infochemicals from spider mites and from food-plants in prey location of the generalist predatory mite Neoseiulus californicus. J. Chem. Ecol. 31:20192032.

Shiojiri, K., Kishimoto, K., OzaWA, R., Kugimiya, S., Urashimo, S., Arimura, G., Horiuchi, J., Nishioka, T., Matsui, K., and TAKABAYASHI, J. 2006a. Changing green leaf volatile biosynthesis in plants: An approach for improving plant resistance against both herbivores and pathogens. Proc. Natl. Acad. Sci. USA 103:16672-16676.

Shiojiri, K., OzaWa, R., Matsui, K., Kishimoto, K., KugimiYa, S., and TAKABAYASHI, J. 2006b. Role of the lipoxygenase/lyase pathway of host-food plants in the host searching behavior of two parasitoid species, Cotesia glomerata and Cotesia plutellae. J. Chem. Ecol. 32:969-979.

StePpuHN, A. and BALDWIN, I. T. 2008. Induced defenses and the cost-benfit paradigm, pp. 61-83, in A. Schaller (ed.). Induced Plant Resistance to Herbivory. Springer Science Business Media B.V, Stuttgart.

StInTZI, A. and BRowse, J. 2000. The Arabidopsis male-sterile mutant, opr3, lacks the 12- oxophytodienoic acid reductase required for jasmonate synthesis. Proc. Natl. Acad. Sci. USA 97:10625-10630.

Stintzi, A., Weber, H., Reymond, P., Browse, J., and Farmer, E. E. 2001. Plant defense in the absence of jasmonic acid: The role of cyclopentenones. Proc. Natl. Acad. Sci. USA 98:12837-12842.

TAKABAYASHI, J. and DICKE, M. 1992. Response of predatory mites with different rearing histories to volatiles of uninfested plants. Entomol. Exp. Appl. 64:187-193.

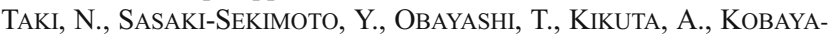
SHI, K., AINAI, T., YAGI, K., SAKURAI, N., SUZUKI, H., MASUdA, T. et al. 2005. 12-oxo-phytodienoic acid triggers expression of a distinct set of genes and plays a role in wound-induced gene expression in Arabidopsis. Plant Physiol. 139:1268-1283.
Thaler, J. S., Farag, M. A., Paré, P. W., and Dicke, M. 2002. Jasmonate-deficient plants have reduced direct and indirect defences against herbivores. Ecol. Lett. 5:764-774.

VAN PoECKE, R. M. P. 2002. Indirect defence of Arabidopsis against herbivorous insects: Combining parasitoid behaviour and chemical analysis with a molecular genetic approach $\mathrm{PhD}$ dissertation.

VAN Poecke, R. M. P. and Dicke, M. 2002. Induced parasitoid attraction by Arabidopsis thaliana: involvement of the octadecanoid and the salicylic acid pathway. J. Exp. Bot. 53:1793-1799.

VAn Poecke, R. M. P., SATO, M., Lenarz-Wyatt, L., Weisberg, S., and KATAGIRI, F. 2007. Natural variation in RPS2-mediated resistance among Arabidopsis accessions: Correlation between gene expression profiles and phenotypic responses. Plant Cell 19:4046-4060.

Vet, L. E. M. and DiCKE, M. 1992. Ecology of infochemical use by natural enemies in a tritrophic context. Annu. Rev. Entomol. No. $37: 141-172$

VoELCKEL, C. and BALDwIN, I. T. 2004. Generalist and specialist lepidopteran larvae elicit different transcriptional responses in Nicotiana attenuata, which correlate with larval FAC profiles. Ecol. Lett. 7:770-775.

Vollenweider, S., Weber, H., Stolz, S., Chetelat, A., and FARMER, E. E. 2000. Fatty acid ketodienes and fatty acid ketotrienes: Michael addition acceptors that accumulate in wounded and diseased Arabidopsis leaves. Plant J. 24:467-476.

Von MaleK, B., VAn Der, GraAfF, E., Schneitz, K., and Keller, B. 2002. The Arabidopsis male-sterile mutant dde2-2 is defective in the ALLENE OXIDE SYNTHASE gene encoding one of the key enzymes of the jasmonic acid biosynthesis pathway. Planta 216:187-192.

WÄCKERS, F. L., ZUBER, D., Wunderlin, R., and Keller, F. 2001. The effect of herbivory on temporal and spatial dynamics of foliar nectar production in cotton and castor. Ann. Bot. 87:365370.

Walling, L. L. 2000. The myriad plant responses to herbivores. $J$. Plant Growth Regul. 19:195-216.

Weber, H., Vick, B. A., and FArmer, E. E. 1997. Dinor-oxophytodienoic acid: A new hexadecanoid signal in the jasmonate family. Proc. Natl. Acad. Sci. USA 94:10473-10478.

Wittstock, U. and Gershenzon, J. 2002. Constitutive plant toxins and their role in defense against herbivores and pathogens. Curr. Opin. Plant Biol. 5:300-307.

Ziegler, J., Hamberg, M., Miersch, O., and Parthier, B. 1997. Purification and characterization of allene oxide cyclase from dry corn seeds. Plant Physiol. 114:565-573. 